\title{
The hexosamine biosynthesis inhibitor azaserine prevents endothelial inflammation and dysfunction under hyperglycemic condition through
} antioxidant effects

\author{
Angana Gupta Rajapakse, Xiu-Fen Ming, João M. Carvas, and Zhihong Yang \\ Division of Physiology, Department of Medicine, Faculty of Science, University of Fribourg, Fribourg, Switzerland
}

\begin{abstract}
Rajapakse AG, Ming XF, Carvas JM, Yang Z. The hexosamine biosynthesis inhibitor azaserine prevents endothelial inflammation and dysfunction under hyperglycemic condition through antioxidant effects. Am J Physiol Heart Circ Physiol 296: H815-H822, 2009. First published January 9, 2009; doi:10.1152/ajpheart.00756.2008.Hexosamine biosynthetic pathway (HBP) accounts for some cardiovascular adverse effects of hyperglycemia. We investigated whether the HBP inhibitor azaserine protects against hyperglycemia-induced endothelial damage dependently of HBP. Human endothelial cells isolated from umbilical veins were exposed either to a high $(30.5$ $\mathrm{mmol} / \mathrm{l})$ or low concentration of glucose $(5.5 \mathrm{mmol} / \mathrm{l})$ for 4 days, followed by a stimulation with TNF- $\alpha$ ( $1 \mathrm{ng} / \mathrm{ml}, 24 \mathrm{~h})$. The blockade of the rate-limiting enzyme glutamine:fructose-6-phosphate amidotransferase inhibited HBP flux and oxidative stress (generation of superoxide and peroxynitrite) under the hyperglycemic condition and prevented the synergistic stimulation of VCAM-1 and ICAM-1 expression by hyperglycemia and TNF- $\alpha$. In the cells cultured under a low-glucose condition when no increased HBP flux occurred, azaserine enhanced the manganese-superoxide dismutase (MnSOD) protein level and also inhibited the oxidative stress and the expression of VCAM-1 and ICAM-1 in response to TNF- $\alpha$. Moreover, the polyphenol resveratrol inhibited the oxidative stress and adhesion molecule expression and did not decrease the HBP flux under the hyperglycemia condition. In addition, in isolated rat aortas exposed to hyperglycemic buffer for $5 \mathrm{~h}$ when no significant HBP flux occurred, azaserine upregulated the MnSOD protein level and prevented decreased endothelium-dependent relaxations to acetylcholine. In conclusion, hyperglycemia independently increases oxidative stress and HBP flux, amplifies endothelial inflammation, and impairs endothelial function mainly through oxidative stress and not the HBP pathway. Azaserine protects against hyperglycemic endothelial damage through its antioxidant effect independently of inhibiting HBP pathway.
\end{abstract}

adhesion molecules; endothelium; glucose; $O$-linked acetylglusamine; superoxide

DIABETES MELLITUS is highly associated with an increased risk of cardiovascular morbidity and mortality $(10,34)$. Chronic hyperglycemia causes glucotoxicity to vascular cells, particularly the endothelial cells, resulting in an increased incidence of cardiovascular events (7). Clinical and experimental studies provide compelling evidence showing that hyperglycemia is associated with impaired endothelium-dependent relaxations (7) and increased endothelial expression of adhesion molecules, vascular cell adhesion molecule-1 (VCAM-1), and intercellular adhesion molecule-1 (ICAM-1) $(20,31)$. The decreased endothelial nitric oxide (NO) synthase (eNOS) func-

Address for reprint requests and other correspondence: Z. Yang, Lab. of Vascular Biology, Dept. of Medicine, Div. of Physiology, Univ. of Fribourg, Rue du Musée 5, CH-1700 Fribourg, Switzerland (e-mail: zhihong.yang @unifr.ch). tion and upregulation of the adhesion molecules enhance the adhesion of inflammatory cells onto the endothelium followed by a transmigration of the cells into the subendothelial space, a key early process in atherogenesis (15). Numerous proinflammatory cytokines such as tumor necrosis factor- $\alpha$ (TNF$\alpha$ ), interleukins, etc., are found to be increased in obesity and obesity-associated metabolic disorders (24). This proinflammatory status causes vascular endothelial inflammation, i.e., an increased expression of VCAM-1 and ICAM-1 (32). It is conceivable that hyperglycemia and proinflammatory cytokines, e.g., TNF- $\alpha$, work in concert to accelerate the vascular endothelial damage, leading to cardiovascular disease.

There is overwhelming evidence demonstrating that hyperglycemia produces superoxide from various sources including mitochondrion, NADPH oxidase, and eNOS uncoupling, which plays a central role in endothelial damage $(4,7,30)$, The increased oxidative stress may be the causal link between various pathways/mechanisms of hyperglycemic endothelial dysfunction. Among them, the increased flux of hexosamine biosynthetic pathway (HBP), which shunts excessive intracellular glucose into the biosynthesis of UDP- $N$-acetylglucosamine (UDP-GlcNAc), has attracted much attention because of its properties to interfere with various cellular functions, including signal transductions, transcriptional activity, cell proliferation, apoptosis, and proteasomal degradation (1, 13, 22). In the HBP pathway, glucose under the sustained hyperglycemic condition is taken up by the cells via glucose transporters and converted to glucosamine-6-phosphate by the rate-limiting enzyme L-glutamine:D-fructose-6-phosphate amidotransferase (GFAT). Glucosamine-6-phosphate is further metabolized to UDP-GlcNAc that serves as the major substrate for the formation of $O$-linked glycoproteins $(O$-GlcNAc) at serine and threonine residues catalyzed by $O$-glycosyltransferase. The increased GFAT activity and $O$-GlcNAc levels are implicated in insulin resistance and type II diabetes mellitus $(16,37)$, associated with increased oxidative stress and, reciprocally, with Akt/eNOS phosphorylation in the endothelial cells, resulting in a decreased endothelial NO production $(11,13,27)$.

Although the increased HBP flux may explain at least in part the decreased eNOS activity, it is, however, not known whether it is involved in endothelial inflammation under the hyperglycemic condition. The relationship between the HBP pathway and oxidative stress seems obscure. It has been reported that oxidative stress stimulates the HBP pathway on one hand (4) and that the HBP pathway is also able to induce 
oxidative stress on the other hand $(19,36)$. Moreover, azaserine is frequently used as an inhibitor of the HBP pathway to delineate its functional roles based on the effect of the compound on cellular $O$-GlcNAc levels. Whether azaserine, like any other chemical substances, could affect cellular functions independently of the HBP pathway is not known. The present study is aimed to investigate the roles of oxidative stress and the HBP pathway in a high glucose (HG)-induced amplification of endothelial inflammatory responses and endothelial damage. The pharmacological mechanisms of azaserine on endothelial protection under the hyperglycemic condition were investigated.

\section{MATERIALS AND METHODS}

Reagents and antibodies. All chemicals, including those for immunoblotting, were obtained from Sigma (Buchs, Switzerland), unless otherwise indicated: L-norepinephrine bitartrate and acetylcholine. TNF- $\alpha$ was purchased from R\&D Systems; dihydroethidium (DHE) and MitoSox were purchased from Invitrogen (Lucerne, Switzerland); antibodies against ICAM-1 and VCAM-1 were from Santa Cruz Biotechnology (Munningen, Switzerland); anti-nitrotyrosine antibody was from Upstate Biotechnol (Luzernachem, Luzern, Switzerland); anti-MnSOD antibody was from Abcam (Cambridge, UK); CTD110.6 antibody was a kind gift from Dr. Gerald W. Hart (The Johns Hopkins University School of Medicine, Baltimore, Maryland); biotin-labeled anti-mouse IgM was purchased from Sigma; Alexa fluor 680-conjugated streptavidin was from Invitrogen; anti- $O$-linked $\mathrm{N}$-acetylglucosamine (RL2) antibody was from Affinity Bioreagents (Lucerne, Switzerland), IRDye 800-conjugated affinity purified goat anti-rabbit IgG $F_{c}$ was purchased from BioConcept (Allschwil, Switzerland); Alexa fluor 680-conjugated goat anti-mouse $\operatorname{IgG}(\mathrm{H}+\mathrm{L})$ was from Invitrogen. Endothelial cell growth supplement pack was from PromoCell (Allschwil, Switzerland), and all cell culture media and materials were purchased from GIBCO (Lucerne, Switzerland).

Endothelial cell culture. Endothelial cells were isolated from human umbilical veins and characterized as described in the study of Ming et al. (26). Cells from the second to fourth passages were used. The endothelial cells were cultured in low-glucose (LG, $5.5 \mathrm{mmol} / \mathrm{l})$ or HG (30.5 mmol/l) DMEM supplemented with 5\% FCS, endothelial cell growth supplement, and $0.6 \mathrm{nmol} / \mathrm{l}$ insulin for 4 days. The cells were then cultured in the same medium, however, supplemented with $1 \%$ FCS for $5 \mathrm{~h}$ followed by the stimulation with TNF- $\alpha(1 \mathrm{ng} / \mathrm{ml})$ for $24 \mathrm{~h}$. To test whether changes in osmolality could contribute to endothelial responses under the HG condition, $25 \mathrm{mmol} / \mathrm{l}$ mannitol were included in the LG medium $(5.5 \mathrm{mmol} / \mathrm{l})$. For some experiments, the cells were incubated with azaserine $(0.2$ to $1 \mu \mathrm{mol} / \mathrm{l})$ or resveratrol (10 $\mu \mathrm{mol} / \mathrm{l})$ throughout the experiments. The same amount of DMSO (used to dissolve resveratrol) was added to the control cells.

Animals. The experimental protocols with animals were approved by the Federal Veterinary Office of Switzerland (Nr. 174/07). WistarKyoto male rats ( $3 \mathrm{mo}$, Harlan) were fed normal chow and maintained according to the local rules of animal experimentation. The animals were anesthetized with pentobarbital sodium $(50 \mathrm{mg} / \mathrm{kg}$ body wt ip) and euthanized. The aortas were removed and cleaned of perivascular fat tissues and prepared for organ chamber experiments.

Endothelium-dependent and -independent relaxations. The aortic rings ( $3 \mathrm{~mm}$ in length) with intact endothelium were prepared as previously described (25). They were suspended in a modified KrebsRinger bicarbonate solution containing (in mmol/l) $118 \mathrm{NaCl}, 4.7$ $\mathrm{KCl}, 2.5 \mathrm{CaCl}_{2}, 1.2 \mathrm{MgSO}_{4}, 1.2 \mathrm{KH}_{2} \mathrm{PO}_{4}, 25 \mathrm{NaHCO}_{3}, 0.026$ EDTA, and 5.5 glucose, aerated with $95 \% \mathrm{O}_{2}-5 \% \mathrm{CO}_{2}$ at $37^{\circ} \mathrm{C}$ in a MultiMyograph System (Model 610M, Danish Myo Technology). One ring served as a control; the second ring was incubated in Krebs buffer containing $25 \mathrm{mmol} / \mathrm{l} \mathrm{D}$-glucose for $5 \mathrm{~h}$, and the third ring in the HG buffer but with $1 \mu \mathrm{mol} / \mathrm{l}$ azaserine. The aortic rings were contracted with norepinephrine $(0.3 \mu \mathrm{mol} / \mathrm{l})$. Endothelium-dependent relaxations were examined with acetylcholine. At the end of the experiments, the aortic rings were snap frozen in liquid nitrogen and kept in $-80^{\circ} \mathrm{C}$ until use.

ICAM-1 and VCAM-1 protein expression and O-linked glycosylation. Cell lysate preparation, $8 \%$ SDS-PAGE, and protein transfer from SDS-PAGE gels to an Immobilon-P membrane (Millipore) were performed as previously described (26). The resultant membranes were first incubated with the individual primary antibody at room temperature for 2 to $3 \mathrm{~h}$ after blocking with $5 \%$ skimmed milk. The blots were then incubated with a corresponding anti-mouse (Alexa fluor 680 conjugated) or anti-rabbit (IRDye 800 conjugated) secondary antibody $(1: 5,000)$ for $2 \mathrm{~h}$, and the quantification of the signals was performed using the Odyssey Application Software 1.2.

For analysis of the $O$-GlcNAc level, the same experimental procedure as described above was used, except that the membranes were first incubated with the mouse antibody against $O$-GlcNAc (RL2) at a dilution of $1: 1,000$ for 2 to $3 \mathrm{~h}$. Alternatively, the membranes were incubated with the antibody CTD110.6 (mouse IgM) at a dilution of 1:2,500 for $2 \mathrm{~h}$ after $1 \mathrm{~h}$ blocking with $3 \%$ bovine serum albumin/ Tris-buffered saline $+0.05 \%$ Tween-20 (BSA/TBST) at room temperature according to the described protocol (6) with slight modifications. The blots were washed three times for 10 min with the TBST, followed by an incubation with biotin-labeled anti-mouse $\operatorname{IgM}$ as a secondary antibody $(1: 10,000)$. After being washed three times with TBST, the blots were finally incubated with Alexa fluor 680-conjugated streptavidin $(1: 5,000)$ for $1 \mathrm{~h}$. The signals were visualized using Odyssey Infrared Imaging System (LI-COR Biosciences, Bad Homburg, Germany). In another series of experiments, aortic tissues were homogenized in ice-cold extraction buffer containing (in mmol/l) 120 $\mathrm{NaCl}, 50$ Tris (pH 8.0), $20 \mathrm{NaF}, 1$ benzamidine, 1 EDTA, 1 EGTA, 1 sodium pyrophosphate, 30 4-nitrophenyl phosphate disodium salt hexahydrate, $1 \%$ Nonidet P-40, and 100 phenylmethylsulfonyl fluoride (PMSF) for immunoblotting to detect $O$-GlcNAc levels as described above.

DHE and MitoSox staining for superoxide anion generation. The endothelial cells were incubated either with $5.0 \mu \mathrm{mol} / \mathrm{l}$ of DHE for 20 min or with MitoSox $(5 \mu \mathrm{mol} / \mathrm{l})$ for $10 \mathrm{~min}$ followed by TNF- $\alpha$ (1 $\mathrm{ng} / \mathrm{ml}$ ) stimulation for $10 \mathrm{~min}$ as described (40). Images were obtained with Zeiss fluorescence microscopy. The intensity of the fluorescence was quantified by ImageJ software (Leica).

Confocal microscopy for immunostaining of 3-nitrotyrosine. Endothelial cells were cultured on coverslips as described for 4 days under the LG and HG condition, respectively, followed by TNF- $\alpha$ stimulation $(1 \mathrm{ng} / \mathrm{ml}, 24 \mathrm{~h})$. The cells were fixed in $4 \%$ paraformaldehyde for $40 \mathrm{~min}$ at room temperature. After being rinsed with PBS for $5 \mathrm{~min}$, the cells were permeabilized with $0.3 \%$ Triton-X/PBS for $10 \mathrm{~min}$ at room temperature. The cells were rinsed three times for $5 \mathrm{~min}$ in PBS, followed by the blocking of nonspecific antigens with $2 \%$ BSA-PBS for $30 \mathrm{~min}$ at room temperature. The cells were then incubated with the anti-nitrotyrosine antibody (1:50) in PBS containing $10 \%$ goat serum for $2 \mathrm{~h}$ at room temperature. The cells were washed three times for $5 \mathrm{~min}$ in PBS and then incubated with Alexa fluor 594-conjugated goat anti-rabbit secondary antibody (1:400) for $1 \mathrm{~h}$ at room temperature. After three times of being rinsed for $5 \mathrm{~min}$, the cells were incubated with 4,6-diamidino-2-phenylindole for $2 \mathrm{~min}$ to stain the nuclei. The cells were finally washed with PBS three times for $5 \mathrm{~min}$ and mounted with anti-fading Vectashield and sealed with nail polish. The sections were then observed for immunofluorescence under a LEICA SP5 confocal microscope. Pictures were taken with a $\times 63$ objective.

FACS analysis of peroxynitrite formation. Endothelial cells were cultured as described for 5 days under the LG and HG condition, respectively. Subsequently, the cells were collected by trypsinization, fixed in $4 \%$ paraformaldehyde, and stained for 3-nitrotyrosine as described in Confocal microscopy for immunostaining of 3-nitrotyrosine. The cells were finally washed three times in PBS, and measure- 
ments were performed using FACS caliber system (PARTEC). Nitrotyrosine was excited by laser at $488 \mathrm{~nm}$, and the data were collected at $590 \mathrm{~nm}$ (FL2) channels. Twenty thousand events were measured, and the data were analyzed by WinMDI software and presented by histogram as the fold increase in the counts of nitrotyrosine positive cells in the different samples.

Statistical analysis. All data were given as means \pm SE. The ANOVA with Bonferroni post hoc test was used for statistical analysis. A two-tailed value of $P<0.05$ was considered to indicate a statistically significant difference.

\section{RESULTS}

Azaserine inhibits endothelial inflammation induced by $H G /$ $T N F-\alpha$. The incubation of the endothelial cells to a HG concentration $(30.5 \mathrm{mmol} / \mathrm{l})$ for 5 days to mimic chronic hyperglycemia did not significantly affect the basal protein level of VCAM-1 or ICAM-1 compared with those of the cells cultured at the LG concentration (5.5 mmol/1, Fig. $1, A$ and $B ; n=5)$; it however, markedly enhanced the stimulating effects of TNF- $\alpha$ (1 ng/ml, $24 \mathrm{~h}$ ) on both VCAM-1 and ICAM-1 expression in the cells (Fig. $1, A$ and $B ; 2.2-$ and 2.5-fold, respectively, $P<0.001$ vs. LG + TNF- $\alpha, n=5$ ). In contrast to glucose, the incubation of the cells with mannitol $(25 \mathrm{mmol} / \mathrm{l})$

A

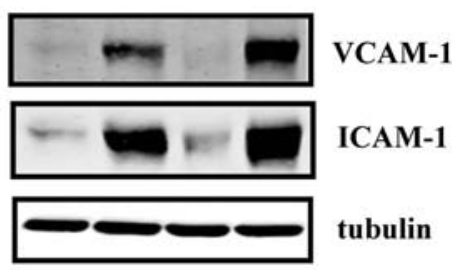

B
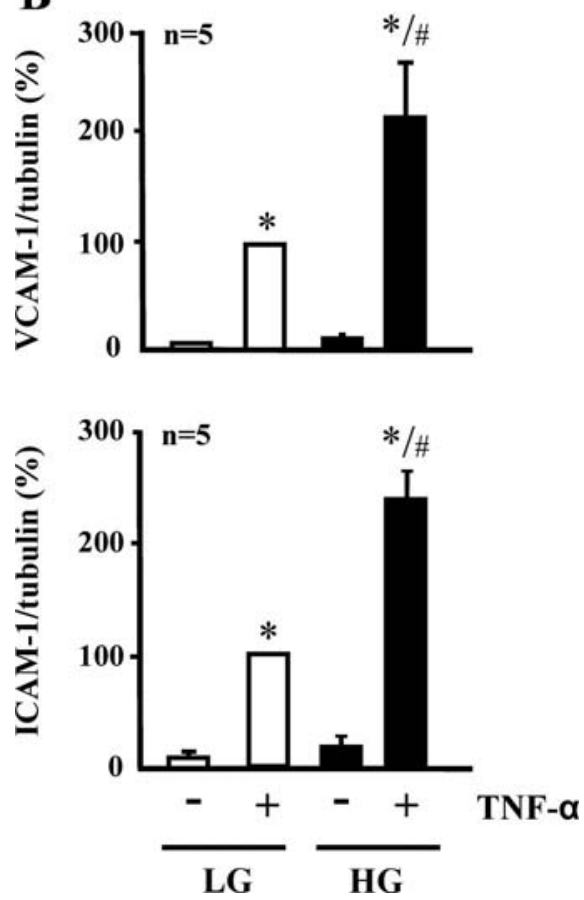

Fig. 1. High glucose (HG) amplifies endothelial adhesion molecule expression to TNF- $\alpha$. A: HG (30.5 mmol/l, 5 days) alone did not significantly affect VCAM-1 and ICAM-1 expression in the endothelial cells, however, but markedly enhanced the effects of TNF- $\alpha(1 \mathrm{ng} / \mathrm{ml})$ on the cell adhesion molecule expression ( $n=5$ sets of experiments). $B$ : quantification of the above results. ${ }^{*} P<0.001$ vs. low glucose (LG); $\# P<0.05$ vs. $\mathrm{LG}+\mathrm{TNF}-\alpha$.

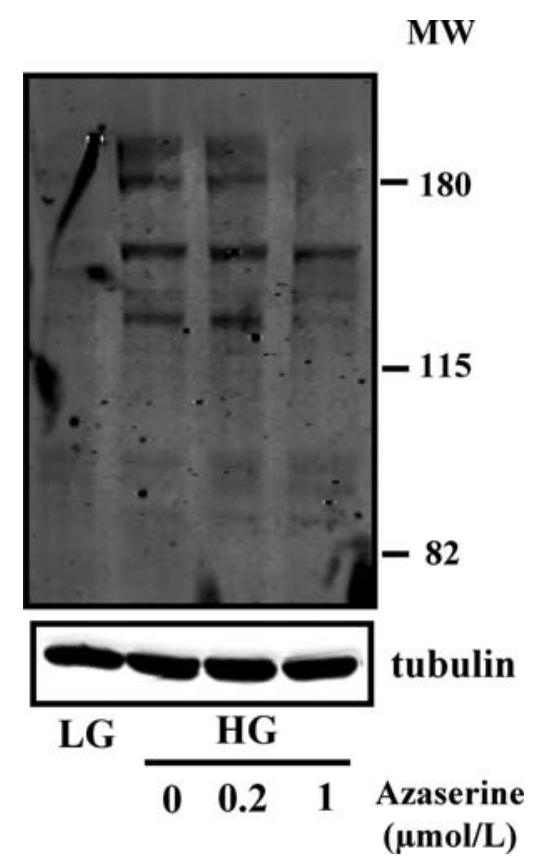

Fig. 2. Azaserine inhibits HG-induced $O$-GlcNAc levels. The increased protein $O$-GlcNAc levels under the $\mathrm{HG}(30.5 \mathrm{mmol} / \mathrm{l}, 5$ days $)$ condition were inhibited by azaserine $(0.2$ to $1.0 \mu \mathrm{mol} / \mathrm{l})$. This experiment was repeated 5 times independently. MW, molecular weight.

in $5.5 \mathrm{mmol} / \mathrm{l} \mathrm{LG}$ medium, to match the osmolality of $\mathrm{HG}$ medium, had no potentiating effects on the TNF- $\alpha$-stimulated levels of VCAM-1 and ICAM-1 (supplemental Fig. 1; note: all supplemental figures may be found posted with the online version of this article).

In parallel, $\mathrm{HG}(30.5 \mathrm{mmol} / \mathrm{l}, 5$ days $)$ enhanced the $O$ GlcNAc modification of various cellular proteins with molecular mass ranging from 115 to $200 \mathrm{kDa}$ (supplemental Fig. 2; $n=4)$. The level of protein $O$-GlcNAc was not affected by TNF- $\alpha$ (supplemental Fig. $2 ; n=4$ ). In the presence of the GFAT inhibitor azaserine $(0.2$ to $1 \mu \mathrm{mol} / \mathrm{l})$, the HG-induced $O$-GlcNAc was significantly reduced (Fig. $2 ; n=5$ ), and the protein levels of both VCAM-1 and ICAM-1 stimulated by TNF- $\alpha$ (1 ng/ml, $24 \mathrm{~h})$ under the $\mathrm{HG}(30.5 \mathrm{mmol} / \mathrm{l})$ condition were also strongly inhibited by azaserine $(0.2$ to $1 \mu \mathrm{mol} / 1$, Fig. 3 ; $n=5, P<0.01-0.001$ vs. HG + TNF- $\alpha)$. Similar inhibitory effects of azaserine were also observed in cells treated with TNF- $\alpha(1 \mathrm{ng} / \mathrm{ml}, 24 \mathrm{~h})$ under the LG $(5.5 \mathrm{mmol} / \mathrm{l})$ condition (Fig. $4 ; n=5, P<0.01-0.001$ vs. TNF- $\alpha$ alone), where the level of protein $O$-GlcNAc was not affected (supplemental Fig. $3 B ; n=3)$. The results suggest that azaserine inhibits endothelial VCAM-1 and ICAM-1 expression through HBP-independent mechanism(s).

Azaserine inhibits endothelial inflammation through antioxidant effects. Superoxide anion generation measured by fluorescence density of DHE staining was increased in the endothelial cells exposed to $\mathrm{HG}$ (30.5 mmol/1, 5 days) medium or $\mathrm{TNF}-\alpha$ alone (1 ng/ml, $10 \mathrm{~min}$ ) and was significantly enhanced when the cells were stimulated by both $\mathrm{HG}$ and TNF- $\alpha$ together (Fig. 5, $A$ and $B$ ). This effect was prevented by azaserine $(0.2$ to $1 \mu \mathrm{mol} / \mathrm{l}$, Fig. $5, A$ and $B ; n=3, P<$ 0.01-0.001 vs. HG + TNF- $\alpha$ ). The antioxidant effect of azaserine was also observed in cells stimulated by TNF- $\alpha$ (1 
A

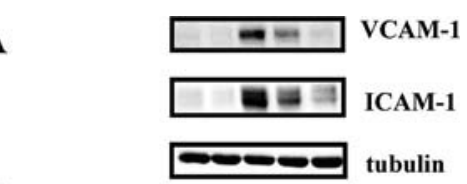

B
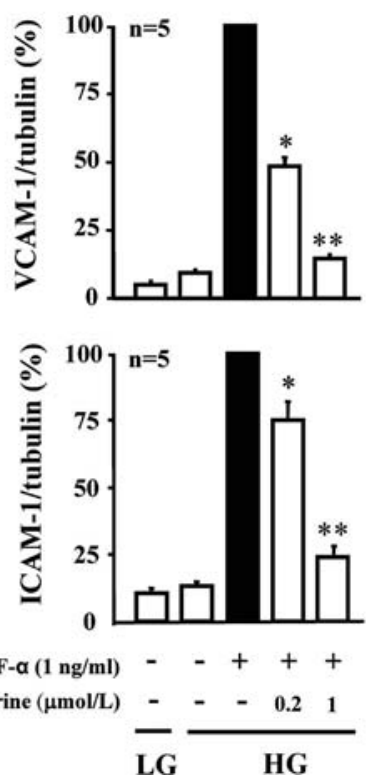

Fig. 3. Azaserine inhibits endothelial adhesion molecule expression under HG condition. A: TNF- $\alpha$-stimulated expression of VCAM-1 and ICAM-1 under HG $(30.5 \mathrm{mmol} / \mathrm{l})$ was inhibited by azaserine ( $n=5$ sets of experiments). $B$ : quantification of the above results. ${ }^{*} P<0.01$ and ${ }^{*} P P<0.001$ vs. HG + TNF- $\alpha$.

$\mathrm{ng} / \mathrm{ml}, 10 \mathrm{~min}$ ) under the LG condition (supplemental Fig. 3A; $n=3$ ) when no increased $O$-GlcNAc occurred (supplemental Fig. $3 B ; n=3$ ). The superoxide anion generation measured by fluorescence density of MitoSox staining was increased in the endothelial cells exposed to $\mathrm{HG}$ (30.5 mmol/1, 5 days) medium, and TNF- $\alpha$ did not further enhance the MitoSox signal. Azaserine $(1 \mu \mathrm{mol} / \mathrm{l})$ was able to inhibit mitochondrial superoxide generation in cells stimulated by HG plus TNF- $\alpha$ (supplemental Fig. 4; $P<0.001, n=4$ ).

Furthermore, an enhanced peroxynitrite formation (cellular 3-nitrotyrosine level) as detected by the confocal microscope was observed under the HG condition or TNF- $\alpha$ stimulation and further increased when the cells were stimulated by HG and TNF- $\alpha$ together (Fig. 6A; $n=4$ ). Quantitative FACS analysis showed an increased peroxynitrite production in cells under the $\mathrm{HG}$ condition, which was further enhanced by TNF- $\alpha$ (Fig. 6, $B$ and $C ; n=4$ ). The stimulation of peroxynitrite formation was fully blocked in the presence of azaserine (Fig. 6, $B$ and $C ; n=4$ ). The results together with those on $O$-GlcNAc obtained under the LG condition suggest that azaserine inhibits endothelial inflammation through antioxidant effects rather than through the inhibition of $O$-GlcNAc synthesis. Moreover, the antioxidant polyphenol resveratrol (10 $\mu \mathrm{mol} / \mathrm{l})$ reduced the upregulation of VCAM- 1 and ICAM- 1 by HG (30.5 mol/l) and TNF- $\alpha(1 \mathrm{ng} / \mathrm{ml}, 24 \mathrm{~h})$ (supplemental Fig. $5 ; n=4, P<0.01$ vs. HG + TNF- $\alpha$ ) and inhibited superoxide anion generation in parallel (supplemental Fig. $6 ; n=4$ ) but did not decrease the $O$-GlcNAc protein level as detected by the two different antibodies (RL2 or CTD110.6) for $O$-GlcNAc (supplemental Fig. 7, $A$ and $B ; n=3$ ).
Interestingly, the protein level of the antioxidant enzyme manganese-superoxide dismutase (MnSOD) was markedly upregulated by azaserine $(1 \mu \mathrm{mol} / \mathrm{l}, 5$ days $)$ in the endothelial cells under the LG condition (Fig. 7, $A$ and $B$, lane 2, 10-fold increase; $n=4, P<0.001$ vs. LG). TNF- $\alpha(1 \mathrm{ng} / \mathrm{ml}, 24 \mathrm{~h})$ under the LG condition was also able to upregulate the MnSOD level (Fig. 7, $A$ and $B$, lane 3, $n=4, P<0.001$ vs. LG alone), whereas HG had no significant effect on MnSOD expression (Fig. 7, $A$ and $B$, lane 5, $n=4$ ). Interestingly, the effect of TNF- $\alpha$ on MnSOD upregulation was abolished under the HG condition (Fig. 7, $A$ and $B$, lane 7). Of particular importance is the observation that the suppressing effect of TNF- $\alpha$ plus HG on MnSOD (lane 7) was prevented by azaserine ( $1 \mu \mathrm{mol} / \mathrm{l}, 5$ days, Fig. 7, $A$ and $B$, lane $8 ; n=4, P<0.001$ vs. lane 7 ). Moreover, a short-time $(1 \mathrm{~h})$ treatment of the cells with azaserine $(1 \mu \mathrm{mol} / \mathrm{l})$ already significantly upregulated MnSOD expression under the LG condition, although this acute effect is much weaker (1.8-fold increase, supplemental Fig. 8) than the chronic effect. It is noteworthy that TNF- $\alpha$ also acutely upregulated MnSOD in the endothelial cells and that the MnSOD level remained high in the presence of both TNF- $\alpha$ and azaserine (supplemental Fig. 8). The superoxide generation stimulated by TNF- $\alpha(1 \mathrm{ng} / \mathrm{ml}, 30 \mathrm{~min})$ under the LG condition was abolished by a 1-h treatment of the cells with azaserine (1 $\mu \mathrm{mol} / \mathrm{l}$ ) (supplemental Fig. 9; $n=4 ; P<0.05$ vs. TNF- $\alpha$ ).

Azaserine prevents $H G$-induced impairment of endotheliumdependent relaxations. In another series of experiments with isolated Wister-Kyoto rat aortic rings with endothelium contracted with norepinephrine $(0.3 \mu \mathrm{mol} / \mathrm{l})$, the incubation of the blood vessels with $25 \mathrm{mmol} / \mathrm{l}$ of glucose for $5 \mathrm{~h}$ significantly impaired endothelium-dependent relaxations in response to

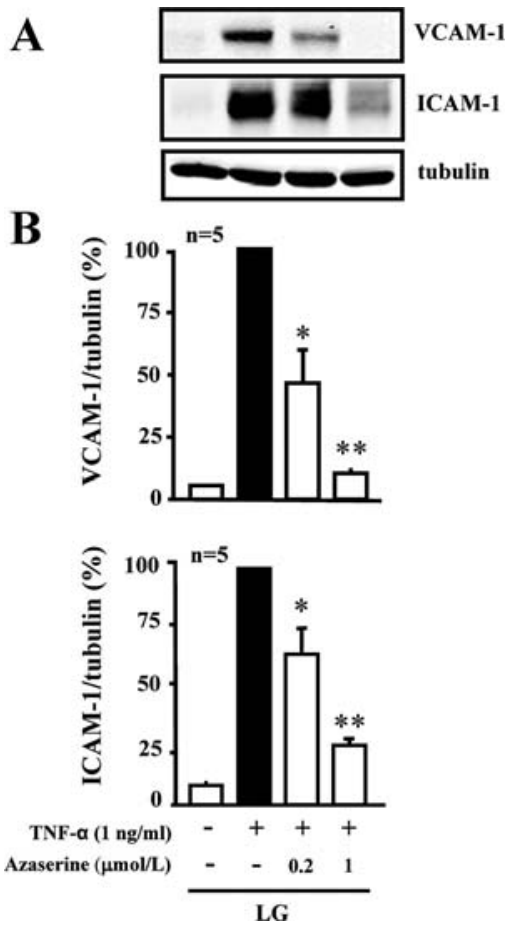

Fig. 4. Azaserine inhibits endothelial adhesion molecule expression under LG conditions. A: TNF- $\alpha$-stimulated expression of VCAM-1 and ICAM-1 under LG $(5.5 \mathrm{mmol} / \mathrm{l})$ was inhibited by azaserine $(n=5$ sets of experiments). $B$ : quantification of the above results. ${ }^{*} P<0.01$ and $* * P<0.001$ vs. TNF- $\alpha$ alone. 


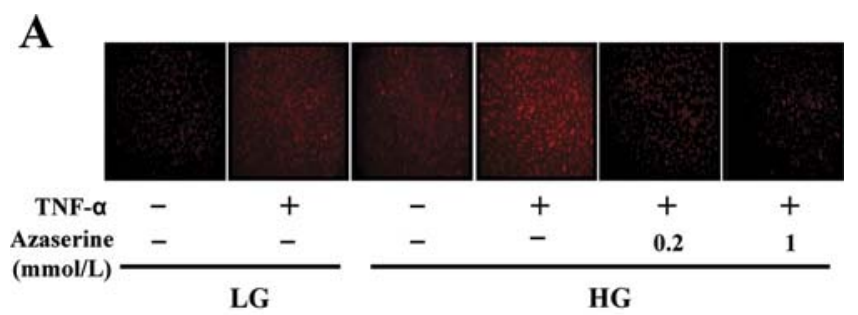

B

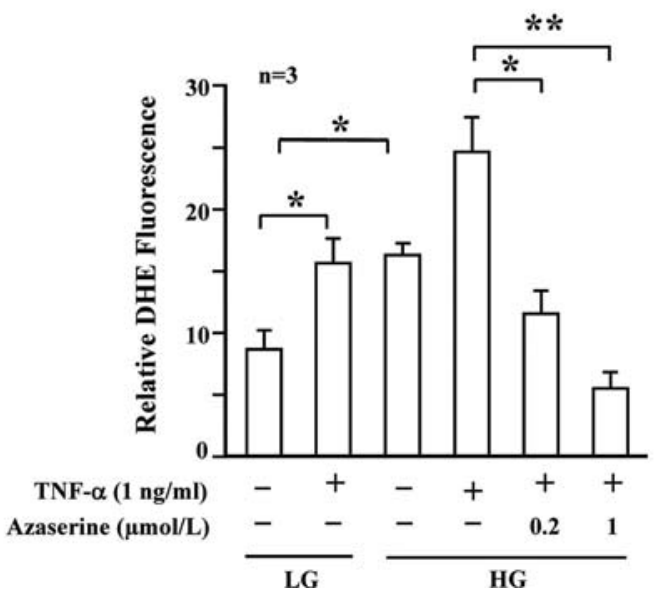

Fig. 5. Azaserine inhibits superoxide generation. A: superoxide generation in human umbilical vein endothelial cells stimulated by TNF- $\alpha$ ( $1 \mathrm{ng} / \mathrm{ml}, 10 \mathrm{~min})$ was enhanced under $\mathrm{HG}$ condition, which was significantly inhibited by azaserine ( 0.2 to $1 \mu \mathrm{mol} / \mathrm{l} ; n=3$ sets of experiments). $B$ : quantification of the above results. DHE, dihydroethidium. $* P<0.05$ and ${ }^{*} * P<0.001$.

acetylcholine ( $1 \mathrm{nmol} / \mathrm{l}$ to $10 \mu \mathrm{mol} / \mathrm{l})($ Fig. $8 A ; n=5)$. This deleterious effect of hyperglycemia on endothelium-dependent relaxations was reversed in the presence of the GFAT inhibitor azaserine $(10 \mu \mathrm{mol} / \mathrm{l}, n=5, P<0.001$ to 0.05 vs. glucose, Fig. 8A). Under the 5-h short-time incubation of aortas in $\mathrm{HG}$ buffer, no significant changes in the $O$-GlcNAc levels in the aortic tissues were observed (Fig. $8 B$, and supplemental Fig. $10 ; n=4)$. Interestingly, in the aortic rings treated with azaserine, the protein level of MnSOD was significantly increased (Fig. $8 C ; n=3, P<0.01$ vs. HG).

\section{DISCUSSION}

Hyperglycemia is proinflammatory and interacts with other inflammatory cytokines. The amplification of the proatherogenic effects in endothelial cells by hyperglycemia has been reported with C-reactive protein $(9,38)$. Our present study further demonstrates that $\mathrm{HG}$ amplifies inflammatory responses of the endothelial cells to the cytokine TNF- $\alpha$. The cells exposed to a HG concentration have no significant effects on the expression of VCAM-1 and ICAM-1 but remarkably enhance the stimulating effects of TNF- $\alpha$. The amplifying effects of HG on the proinflammatory cytokines may facilitate vascular damage, contributing to the higher clinical adverse outcomes in diabetic patients with inflammatory markers (33).

Studies from other groups showed a significant increased expression of the adhesion molecules by $\mathrm{HG}$ exposure in cultured human umbilical vein endothelial cells and human coronary artery endothelial cells $(2,18,29)$, which is slightly different from the results of our present study. We show only a weak but not significant increase in the expression of ICAM-1 and VCAM-1 by HG (e.g., Fig. 1, or supplemental Fig. 4). This slightly different discrepancy might be due to the different experimental conditions. For example, in the study by Altannavch et al. (2), the cells were stimulated by the HG medium containing $20 \%$ serum. An interaction of $\mathrm{HG}$ and serum factors in the stimulation of adhesion molecule expression most likely exists. In other studies $(18,29,31)$, no information is available on how much serum was included in the HG medium for the cells. In contrast, in our current study, the cells were exposed to HG medium containing a very low serum concentration (1\% FCS) to avoid a possible strong interaction between $\mathrm{HG}$ and serum factors for the stimulation
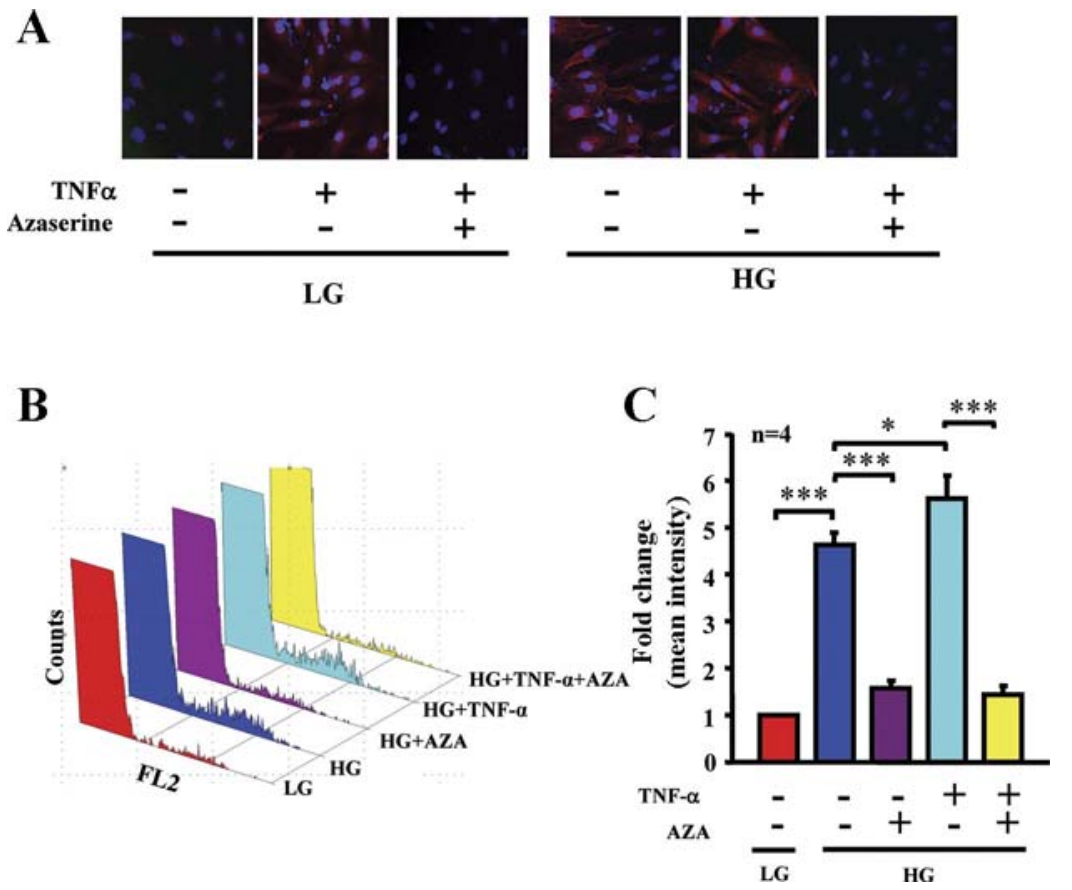

Fig. 6. Azaserine (AZA) inhibits peroxynitrite formation. $A$ : confocal microscopy and FACS analysis $(B$ and $C$ ) demonstrate peroxynitrite generation (3-nitrotyrosine formation) in the endothelial cells stimulated by TNF- $\alpha, \mathrm{HG}$, or both together ( $n=4$ sets of experiments). ${ }^{*} P<0.05$ and ${ }^{*} * * P<0.001$. 
A

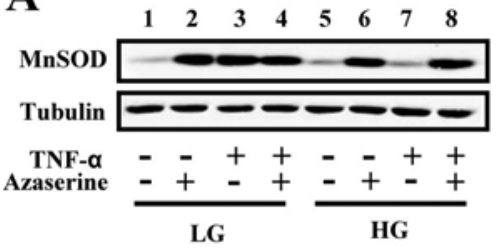

B

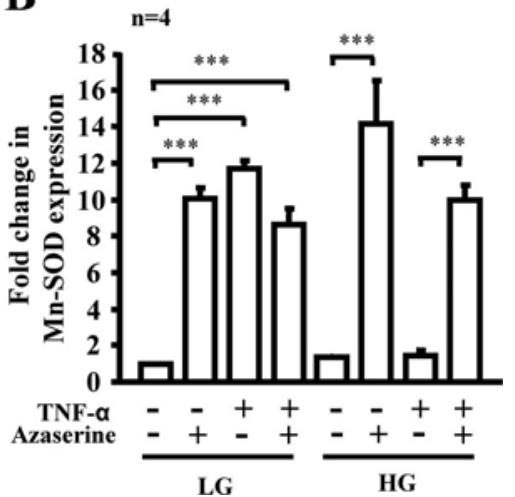

Fig. 7. Effects of azaserine on MnSOD expression in the endothelial cells: $A$ : chronic effects of azaserine: TNF- $\alpha$ (lane 3) not HG (lane 5) upregulates MnSOD expression. The effect of TNF- $\alpha$ is abolished by HG (lane 7). The downregulation of MnSOD by TNF- $\alpha$ under HG condition (lane 7) is reversed by azaserine (lane 8 ). Treatment of cells with azaserine upregulates MnSOD protein level under $\mathrm{LG}$ condition (lane 2). $B$ : quantification of the above results ( $n=4$ sets of experiments). $* * * P<0.001$.

of endothelial adhesion molecule expression while maintaining the cells in a viable but inactivated situation for 5 days. Our data do not show a significant increase in the endothelial expression of the adhesion molecules but demonstrate a potentiating effect on TNF- $\alpha$-induced VCAM- 1 and ICAM- 1 expression. Our in vitro experimental results are in line with the clinical observations showing the synergistic effects of hyperglycemia and inflammation in clinical outcomes of patients with acute coronary syndromes $(9,33,38)$.

The vascular endothelial cell damage caused by hyperglycemia involves metabolic dysfunctions in the cells and oxidative stress $(4,28)$. Among these mechanisms, the increased HBP flux has been reported to account for many of the adverse effects observed in diabetes mellitus, including insulin resis- tance and diabetic vascular complications (16). For example, hyperglycemia induces the $O$-GlcNAc modification of transcription factor Sp1 and increases its activity in aortic endothelial cells, resulting in an enhanced transcription of transforming growth factor- $\beta_{1}$ and plasminogen activator inhibitor-1. Elevated $O$-GlcNAc levels have been observed in coronary endothelial cells under the HG condition and in atherosclerotic plaques from humans with diabetes compared with humans without diabetes (13). eNOS activity in cultured human coronary and bovine endothelial cells is inhibited by hyperglycemia through the $O$-GlcNAc modification and the reciprocal reduction in phosphorylation at Ser1177, contributing to a reduced endothelial-dependent vasodilatation in diabetic patients. Indeed, Musicki et al. (27) demonstrated that diabetes-related erectile dysfunction is associated with a hyperglycemia-induced $O$-GlcNAc modification of eNOS (27). In accordance, we show in the current study that HG increases $O$-GlcNAc levels in the endothelial cells. However, the increased $O$-GlcNAc does not seem to account for the amplifying effect of HG on the TNF- $\alpha$-induced expression of VCAM-1 and ICAM-1. These conclusions are based on the following results. First, $O$-GlcNAc levels induced by HG are not influenced by TNF- $\alpha$. Second, although the GFAT inhibitor azaserine reduces $O$-GlcNAc levels under the HG condition and inhibits VCAM-1 and ICAM-1 expression, these effects of azaserine are also observed when the cells are stimulated with TNF- $\alpha$ under the LG condition, at which no obvious increase in protein modification of $O$-GlcNAc occurs. The results suggest that azaserine inhibits VCAM-1 and ICAM-1 expression mainly through mechanisms that are most likely independent of protein $O$-GlcNAc.

It is well known that oxidative stress plays an important role in endothelial dysfunction under a HG condition, which appears to be the crucial mechanism for the pathogenesis of diabetic vascular complications $(3,30)$. Numerous studies demonstrate that a hyperglycemia-induced superoxide generation can derive from multiple sources in endothelial cells, including mitochondrion, xanthine oxidase, NAD(P)H-oxidase, cyclooxygenase, and uncoupled eNOS (14). The superoxide anion reacts with $\mathrm{NO}$, resulting in a peroxynitrite generation that is a more potent oxidant causing further oxidative damage to the cells (14). There is compelling evidence from recent studies showing that the mitochondrial superoxide gen-

Fig. 8. Azaserine prevents hyperglycemia-induced impairment of endothelium-dependent relaxations. $A$ : incubation of rat aortas with $25 \mathrm{mmol} / \mathrm{l}$ glucose in Krebs buffer for $5 \mathrm{~h}$ decreased the endotheliumdependent relaxations to acetylcholine ( $n=5$ rats), which was prevented in the presence of azaserine (1 $\mu \mathrm{mol} / \mathrm{l}) . P<0.05, * * P<0.01$, and $* * * P<0.005$ vs. control $(5.5 \mathrm{mmol} / \mathrm{l}$ glucose $) . B$ : no changes in $O$-linked $N$-acetylglucosamine $(O$-GlcNAc) levels were observed in the aortas under the condition of short-term incubation in HG buffer ( $n=4$ rats). RL2, antibody against $O$-GlcNAc. $C$ : MnSOD protein level was significantly increased in aortas treated with azaserine $\left(1 \mu \mathrm{mol} / \mathrm{l}, 5 \mathrm{~h}, n=3\right.$ rats). ${ }^{*} * P<0.01$ vs. HG.
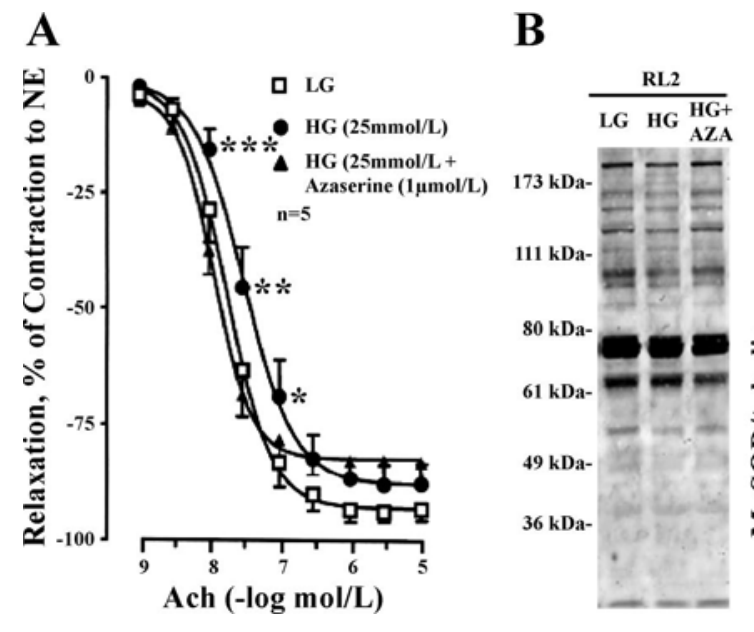

C

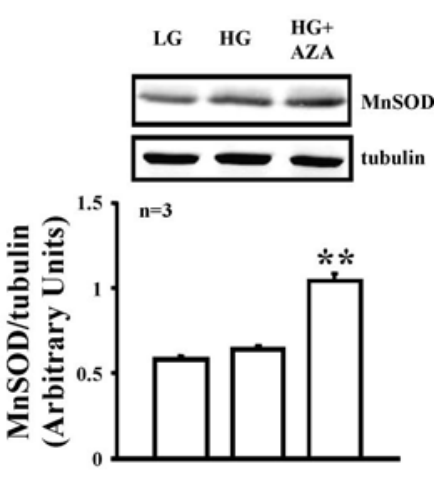


eration plays a major role in oxidative stress under the hyperglycemic condition $(23,28,29,31)$. The mitochondrial superoxide generation has been demonstrated to mediate the upregulation of endothelial adhesion molecule expression (31) and has been shown to be prevented by polyphenol resveratrol (23). With MitoSox as the mitochondrial reactive oxygen species (ROS) indicator, we also show that HG generates superoxide from the mitochondrion that is inhibited by azaserine (supplemental Fig. 4). TNF- $\alpha$ does not further enhance the MitoSox signal, suggesting that TNF- $\alpha$, in contrast to glucose, stimulates superoxide production mainly from other sources than from the mitochondrion. Indeed, studies from other groups demonstrate much higher ROS production from sources other than the mitochondrion in response to TNF- $\alpha$ in the endothelial cells (8). The results from these studies, however, do not exclude the role of the other sources of ROS generation that is stimulated by HG. Moreover, nitrotyrosine formation, the footprint of peroxynitrite resulting from reaction of superoxide anion and NO derived either from uncoupled eNOS or inducible NO synthase (31), is also increased in the cells exposed to HG and further enhanced by TNF- $\alpha$ (Fig. 6), demonstrating the interaction of $\mathrm{HG}$ and TNF- $\alpha$ in oxidative stress. Interestingly, the effects of $\mathrm{HG}$ and TNF- $\alpha$ on both superoxide generation and nitrotyrosine formation, as well as the endothelial expression of adhesion molecules, are inhibited by azaserine, demonstrating that azaserine inhibits ICAM-1 and VCAM-1 expression via the inhibition of oxidative stress rather than the inhibition of the HBP pathway. This conclusion is further supported by the fact that the polyphenol resveratrol that has been shown to exert antioxidant effects (21) inhibits superoxide generation stimulated by HG and TNF- $\alpha$, reduces VCAM- 1 and ICAM-1 expression in parallel (supplemental Fig. 5, and supplemental Fig. 6), but does not decrease the $O$-GlcNAc level induced by HG (supplemental Fig. 7). Finally, the results that azaserine protects against impaired endothelium-dependent relaxations caused by an acute exposure of the aortas to HG (5 h), whereby no changes in $O$-GlcNAc levels in the blood vessels were observed, further support the antioxidant effect of the GFAT inhibitor azaserine (Fig. 8). The antioxidative effect of azaserine has been noted in cultured renal proximal tubular cells (17). Moreover, the results with resveratrol suggest that the inhibition of superoxide generation by resveratrol accounts for the inhibition of endothelial adhesion molecule expression stimulated by HG and TNF- $\alpha$. The results of our current study do not support the hypothesis that hyperglycemia-induced superoxide generation drives $O$-GlcNAc formation (12) but rather suggest that hyperglycemia causes oxidative stress and HBP flux independently. The reason for the disparity remains obscure at this stage.

One of the interesting findings is that the treatment of the endothelial cells with azaserine is able to upregulate MnSOD protein under the LG condition. HG alone does not affect the MnSOD protein level, whereas TNF- $\alpha$ is able to upregulate MnSOD protein expression (Fig. 7), which may reflect the insufficient compensatory effect of the antioxidative system. The upregulation of MnSOD by TNF- $\alpha$ has been also reported by other groups $(35,39)$. The upregulation of MnSOD by TNF- $\alpha$ is diminished under the HG condition. This could be due to the exaggerated oxidative injury to the cells. Interestingly, the downregulation of MnSOD by TNF- $\alpha$ under the HG condition is reversed by azaserine (Fig. 7), suggesting that the antioxidative effect of azaserine may be attributed by the upregulation of MnSOD. Moreover, the upregulation of MnSOD is even observed after a short-time $(1 \mathrm{~h})$ treatment of the cells with azaserine (supplemental Fig. 8), which is associated with the inhibition of superoxide generation (supplemental Fig. 9). Similar to the experiments in cultured cells, the treatment of aortic rings ex vivo with azaserine also increases the MnSOD protein level (Fig. 8C), which is associated with improved endothelium-dependent relaxations (Fig. 8A). Whether the upregulation of MnSOD fully accounts for the antioxidative effect of azaserine requires further investigation. The results demonstrate at least an important mechanism of anti-oxidative effect of azaserine in vascular cells.

In conclusion, hyperglycemia causes oxidative stress and HBP flux, amplifies endothelial inflammation, and impairs endothelial function mainly through oxidative stress and not the HBP pathway. It emphasizes that the results do not exclude the potential role of the HBP pathway in the inhibition of insulin-mediated metabolic signalings in the insulin target tissues and eNOS activation $(5,11,13,41)$. Our results demonstrate that azaserine not only inhibits the HBP pathway but is also a strong antioxidant. Azaserine prevents against endothelial inflammation and the impairment of endotheliumdependent relaxations under the hyperglycemic condition through the inhibition of oxidant stress independently of the inhibiting HBP pathway.

\section{ACKNOWLEDGMENTS}

We thank Jean Ruffieux for technical assistance in isolation of endothelial cells, Kiran Todkar for technical advises for FACS analysis, and Isabelle Scerri for confocal microscopy.

\section{GRANTS}

This study was supported by Swiss National Science Foundation Grants Nr. 3100A0-105917 and 310000-120435/1, the Swiss Heart Foundation, and the Swiss Cardiovascular Research and Training Network Program. J. M. Carvas was partially supported by the Roche Research Foundation.

\section{REFERENCES}

1. Akimoto Y, Kreppel LK, Hirano H, Hart GW. Hyperglycemia and the $O$-GlcNAc transferase in rat aortic smooth muscle cells: elevated expression and altered patterns of $O$-GlcNAcylation. Arch Biochem Biophys 389: $166-175,2001$.

2. Altannavch TS, Roubalova K, Kucera P, Andel M. Effect of high glucose concentrations on expression of ELAM-1, VCAM-1 and ICAM-1 in HUVEC with and without cytokine activation. Physiol Res 53: 77-82, 2004.

3. Brownlee M. The pathobiology of diabetic complications: a unifying mechanism. Diabetes 54: 1615-1625, 2005.

4. Brownlee M. Biochemistry and molecular cell biology of diabetic complications. Nature 414: 813-820, 2001.

5. Buse MG. Hexosamines, insulin resistance, and the complications of diabetes: current status. Am J Physiol Endocrinol Metab 290: E1-E8, 2006.

6. Comer FI, Vosseller K, Wells L, Accavitti MA, Hart GW. Characterization of a mouse monoclonal antibody specific for $O$-linked $N$-acetylglucosamine. Anal Biochem 293: 169-177, 2001.

7. Creager MA, Luscher TF, Cosentino F, Beckman JA. Diabetes and vascular disease: pathophysiology, clinical consequences, and medical therapy: Part I. Circulation 108: 1527-1532, 2003.

8. Deshpande SS, Angkeow P, Huang J, Ozaki M, Irani K. Rac1 inhibits TNF-alpha-induced endothelial cell apoptosis: dual regulation by reactive oxygen species. FASEB $J$ 14: 1705-1714, 2000.

9. Devaraj S, Xu DY, Jialal I. C-reactive protein increases plasminogen activator inhibitor-1 expression and activity in human aortic endothelial cells: implications for the metabolic syndrome and atherothrombosis. Circulation 107: 398-404, 2003. 
10. Donahoe SM, Stewart GC, McCabe CH, Mohanavelu S, Murphy SA, Cannon CP, Antman EM. Diabetes and mortality following acute coronary syndromes. JAMA 298: 765-775, 2007.

11. Du XL, Edelstein D, Dimmeler S, Ju Q, Sui C, Brownlee M. Hyperglycemia inhibits endothelial nitric oxide synthase activity by posttranslational modification at the Akt site. J Clin Invest 108: 1341-1348, 2001.

12. Du XL, Edelstein D, Rossetti L, Fantus IG, Goldberg H, Ziyadeh F, Wu J, Brownlee M. Hyperglycemia-induced mitochondrial superoxide overproduction activates the hexosamine pathway and induces plasminogen activator inhibitor-1 expression by increasing $\mathrm{Sp} 1$ glycosylation. Proc Natl Acad Sci USA 97: 12222-12226, 2000.

13. Federici M, Menghini R, Mauriello A, Hribal ML, Ferrelli F, Lauro D, Sbraccia P, Spagnoli LG, Sesti G, Lauro R. Insulin-dependent activation of endothelial nitric oxide synthase is impaired by $O$-linked glycosylation modification of signaling proteins in human coronary endothelial cells. Circulation 106: 466-472, 2002.

14. Forstermann U. Oxidative stress in vascular disease: causes, defense mechanisms and potential therapies. Nat Clin Pract Cardiovasc Med 5: 338-349, 2008.

15. Galkina E, Ley K. Vascular adhesion molecules in atherosclerosis. Arterioscler Thromb Vasc Biol 27: 2292-2301, 2007.

16. Hart GW, Housley MP, Slawson C. Cycling of $O$-linked beta- $N$-acetylglucosamine on nucleocytoplasmic proteins. Nature 446: 1017-1022, 2007.

17. Hsieh TJ, Fustier P, Wei CC, Zhang SL, Filep JG, Tang SS, Ingelfinger JR, Fantus IG, Hamet P, Chan JS. Reactive oxygen species blockade and action of insulin on expression of angiotensinogen gene in proximal tubular cells. J Endocrinol 183: 535-550, 2004.

18. Itoh M, Omi H, Okouchi M, Imaeda K, Shimizu M, Fukutomi T, Okayama N. The mechanisms of inhibitory actions of gliclazide on neutrophils-endothelial cells adhesion and surface expression of endothelial adhesion molecules mediated by a high glucose concentration. $J$ Diabetes Complications 17: 22-26, 2003.

19. Kaneto H, Xu G, Song KH, Suzuma K, Bonner-Weir S, Sharma A, Weir GC. Activation of the hexosamine pathway leads to deterioration of pancreatic beta-cell function through the induction of oxidative stress. J Biol Chem 276: 31099-31104, 2001.

20. Konukoglu D, Firtina S, Serin $\mathbf{O}$. The relationship between plasma asymmetrical dimethyl-L-arginine and inflammation and adhesion molecule levels in subjects with normal, impaired, and diabetic glucose tolerance. Metabolism 57: 110-115, 2008.

21. Labinskyy N, Csiszar A, Veress G, Stef G, Pacher P, Oroszi G, Wu J, Ungvari Z. Vascular dysfunction in aging: potential effects of resveratrol, an anti-inflammatory phytoestrogen. Curr Med Chem 13: 989-996, 2006

22. Love DC, Hanover JA. The hexosamine signaling pathway: deciphering the "O-GlcNAc code". Sci STKE 2005: re13, 2005.

23. Lu C, Bambang IF, Armstrong JS, Whiteman M. Resveratrol blocks high glucose-induced mitochondrial reactive oxygen species production in bovine aortic endothelial cells: role of phase 2 enzyme induction? Diabetes Obes Metab 10: 347-349, 2008.

24. Lu L, Pu LJ, Xu XW, Zhang Q, Zhang RY, Zhang JS, Hu J, Yang ZK, Lu AK, Ding FH, Shen J, Chen QJ, Lou S, Fang DH, Shen WF. Association of serum levels of glycated albumin, C-reactive protein and tumor necrosis factor-alpha with the severity of coronary artery disease and renal impairment in patients with type 2 diabetes mellitus. Clin Biochem 40: 810-816, 2007.

25. Ming XF, Barandier C, Viswambharan H, Kwak BR, Mach F, Mazzolai L, Hayoz D, Ruffieux J, Rusconi S, Montani JP, Yang Z. Thrombin stimulates human endothelial arginase enzymatic activity via RhoA/ROCK pathway: implications for atherosclerotic endothelial dysfunction. Circulation 110: 3708-3714, 2004

26. Ming XF, Viswambharan H, Barandier C, Ruffieux J, Kaibuchi K, Rusconi S, Yang Z. Rho GTPase/Rho kinase negatively regulates endothelial nitric oxide synthase phosphorylation through the inhibition of protein kinase B/Akt in human endothelial cells. Mol Cell Biol 22: 8467-8477, 2002.

27. Musicki B, Kramer MF, Becker RE, Burnett AL. Inactivation of phosphorylated endothelial nitric oxide synthase (Ser-1177) by $O$-GlcNAc in diabetes-associated erectile dysfunction. Proc Natl Acad Sci USA 102: 11870-11875, 2005.

28. Nishikawa T, Edelstein D, Du XL, Yamagishi S, Matsumura T, Kaneda Y, Yorek MA, Beebe D, Oates PJ, Hammes HP, Giardino I, Brownlee M. Normalizing mitochondrial superoxide production blocks three pathways of hyperglycaemic damage. Nature 404: 787-790, 2000.

29. Quagliaro L, Piconi L, Assaloni R, Da RR, Maier A, Zuodar G, Ceriello A. Intermittent high glucose enhances ICAM-1, VCAM-1 and E-selectin expression in human umbilical vein endothelial cells in culture: the distinct role of protein kinase $\mathrm{C}$ and mitochondrial superoxide production. Atherosclerosis 183: 259-267, 2005.

30. Quijano C, Castro L, Peluffo G, Valez V, Radi R. Enhanced mitochondrial superoxide in hyperglycemic endothelial cells: direct measurements and formation of hydrogen peroxide and peroxynitrite. Am J Physiol Heart Circ Physiol 293: H3404-H3414, 2007.

31. Rajesh M, Mukhopadhyay P, Batkai S, Hasko G, Liaudet L, Drel VR, Obrosova IG, Pacher P. Cannabidiol attenuates high glucose-induced endothelial cell inflammatory response and barrier disruption. Am J Physiol Heart Circ Physiol 293: H610-H619, 2007.

32. Rajesh M, Mukhopadhyay P, Batkai S, Hasko G, Liaudet L, Huffman JW, Csiszar A, Ungvari Z, Mackie K, Chatterjee S, Pacher P. $\mathrm{CB}_{2-}$ receptor stimulation attenuates TNF- $\alpha$-induced human endothelial cell activation, transendothelial migration of monocytes, and monocyte-endothelial adhesion. Am J Physiol Heart Circ Physiol 293: H2210-H2218, 2007.

33. Ray KK, Cannon CP, Morrow DA, Kirtane AJ, Buros J, Rifai N, McCabe CH, Gibson CM, Braunwald E. Synergistic relationship between hyperglycaemia and inflammation with respect to clinical outcomes in non-ST-elevation acute coronary syndromes: analyses from OPUSTIMI 16 and TACTICS-TIMI 18. Eur Heart $J$ 28: 806-813, 2007.

34. Selvin E, Marinopoulos S, Berkenblit G, Rami T, Brancati FL, Powe NR, Golden SH. Meta-analysis: glycosylated hemoglobin and cardiovascular disease in diabetes mellitus. Ann Intern Med 141: 421-431, 2004.

35. Shaffer JB, Treanor CP, Del Vecchio PJ. Expression of bovine and mouse endothelial cell antioxidant enzymes following TNF-alpha exposure. Free Radic Biol Med 8: 497-502, 1990.

36. Singh LP, Cheng DW, Kowluru R, Levi E, Jiang Y. Hexosamine induction of oxidative stress, hypertrophy and laminin expression in renal mesangial cells: effect of the anti-oxidant alpha-lipoic acid. Cell Biochem Funct 25: 537-550, 2007.

37. Srinivasan V, Sandhya N, Sampathkumar R, Farooq S, Mohan V, Balasubramanyam M. Glutamine fructose-6-phosphate amidotransferase (GFAT) gene expression and activity in patients with type 2 diabetes: inter-relationships with hyperglycaemia and oxidative stress. Clin Biochem 40: 952-957, 2007.

38. Verma S, Wang CH, Weisel RD, Badiwala MV, Li SH, Fedak PW, Li RK, Mickle DA. Hyperglycemia potentiates the proatherogenic effects of C-reactive protein: reversal with rosiglitazone. J Mol Cell Cardiol 35: 417-419, 2003.

39. Visner GA, Chesrown SE, Monnier J, Ryan US, Nick HS. Regulation of manganese superoxide dismutase: IL-1 and TNF induction in pulmonary artery and microvascular endothelial cells. Biochem Biophys Res Commun 188: 453-462, 1992.

40. Wedgwood S, McMullan DM, Bekker JM, Fineman JR, Black SM. Role for endothelin-1-induced superoxide and peroxynitrite production in rebound pulmonary hypertension associated with inhaled nitric oxide therapy. Circ Res 89: 357-364, 2001.

41. Yang X, Ongusaha PP, Miles PD, Havstad JC, Zhang F, So WV, Kudlow JE, Michell RH, Olefsky JM, Field SJ, Evans RM. Phosphoinositide signalling links $O$-GlcNAc transferase to insulin resistance. Nature 451: 964-969, 2008. 